\title{
Deep inelastic $e-\tau$ and $\mu-\tau$ conversion in the NA64 experiment at the CERN SPS
}

\author{
Sergei Gninenko, ${ }^{1}$ Sergey Kovalenko, ${ }^{2}$ Serguei Kuleshov, ${ }^{2}$ Valery E. Lyubovitskij, ${ }^{2,3,4,5}$ and Alexey S. Zhevlakov ${ }^{4,6}$ \\ ${ }^{1}$ Institute for Nuclear Research, 117312 Moscow, Russia \\ ${ }^{2}$ Departamento de Física y Centro Científico Tecnológico de Valparaíso-CCTVal, \\ Universidad Técnica Federico Santa María, Casilla 110-V, Valparaíso, Chile \\ ${ }^{3}$ Institut für Theoretische Physik, Universität Tübingen, Kepler Center for Astro and Particle Physics, \\ Auf der Morgenstelle 14, D-72076, Tübingen, Germany \\ ${ }^{4}$ Department of Physics, Tomsk State University, 634050 Tomsk, Russia \\ ${ }^{5}$ Tomsk State Pedagogical University, 634061 Tomsk, Russia \\ ${ }^{6}$ Matrosov Institute for System Dynamics and Control Theory SB RAS, \\ Lermontov str. 134, 664033, Irkutsk, Russia
}

(Received 17 April 2018; published 5 July 2018)

\begin{abstract}
We study the lepton flavor violating (LFV) $e(\mu)-\tau$ conversion in deep inelastic scattering (DIS) of electrons (muons) on fixed-target nuclei. Our model-independent analysis is based on the set of low-energy effective four-fermion LFV operators composed of leptons and quarks with the corresponding mass scales $\Lambda_{k}$ for each operator. Using the estimated sensitivity of the search for this LFV process in events with large missing energy in the NA64 experiment at the CERN SPS, we derive lower limits for $\Lambda_{k}$ and compared them with the corresponding limits existing in the literature. We show that the DIS $e(\mu)-\tau$ conversion is able to provide a plenty of new limits as yet nonexisting in the literature. We also analyzed the energy spectrum of the final-state $\tau$ and discussed the viability of the observation of this process in the NA64 experiment and ones akin to it. The case of polarized beams and targets is also discussed.
\end{abstract}

DOI: 10.1103/PhysRevD.98.015007

\section{INTRODUCTION}

The lepton flavor violation (LFV) is absent in the standard model (SM), if neutrinos are massless. Nowadays, nonzero neutrino masses and flavor mixing is a matter of experimental fact. LFV can be transmitted from the neutrino sector to the charged lepton one via the charged-current neutrino loop which, however, is heavily suppressed by the small neutrino mass square-differences. On the other hand, the possible high-scale physics beyond the SM (BSM) may contribute to the LFV in the charged lepton sector directly without the mediation of the neutrino sector. The high-scale BSM leaks into the low-energy theory via several universal effective nonrenormalizable LFV operators parametrizing in a generic way all the possible UV realization of BSM. In what follows, we specify these operators and estimate their possible contribution to the deep-inelastic $e-\tau$ and $\mu-\tau$ conversion

Published by the American Physical Society under the terms of the Creative Commons Attribution 4.0 International license. Further distribution of this work must maintain attribution to the author(s) and the published article's title, journal citation, and DOI. Funded by SCOAP ${ }^{3}$.

$$
e(\mu)+(A, Z) \rightarrow \tau+X
$$

of the initial electrons (muons) in the fixed-target with the atomic and mass numbers $Z$ and $A$, respectively.

The $e+p \rightarrow \tau+X$ process was searched for by the ZEUS Collaboration at HERA (DESY) [1] in $e^{+} p$ collisions at a center-of-mass energy $\sqrt{s} \simeq 300 \mathrm{GeV}$. Theoretical study of the $e-\tau, \mu-e$, and $\mu-\tau$ conversion has been done before in Refs. [2-24]. The experimental study of $e-\tau$ and $\mu-\tau$ conversion is planned by the NA64 experiment at the CERN SPS. The NA64 experiment is a fixed-target experiment combining the active beam dump and missing energy techniques to search for rare events. The experiment will build and operate a fully hermetic detector placed on the $\mathrm{H} 4$ beam line at the CERN SPS with the primary goal to search for light dark photon $\left(A^{\prime}\right)$ coupled to photon, e.g., dark photons $\left(A^{\prime}\right)$, or sub-GeV dark gauge boson $Z^{\prime}$ coupled only to quarks or only to charged leptons. Other goals of the experiment are to search for the $L_{\mu}-L_{\tau}$ gauge boson and the $K_{L} \rightarrow$ invisible decay, which is complementary to $K^{+} \rightarrow \pi^{+} \nu \bar{\nu}$, and invisible decays of light pseudoscalar mesons $\left(\pi^{0}, \eta, \eta, K_{S}\right)$.

The NA64 experiment is also capable for study lepton conversion in the inclusive scattering of electrons or muons on nuclei $e^{-}\left(\mu^{-}\right)+(A, Z) \rightarrow \tau^{-}+X$. This inclusive 
experimental mode is the only realistic for such kind of processes at the NA64 experimental setup. Note that elastic and quasi-elastic channels are included in deep inelastic cross section we analyzed. For study of the $e-\tau$ conversion the NA64 experiment could employ the $100 \mathrm{GeV}$ electron beam from the $\mathrm{H} 4$ beamline with a maximal intensity $\simeq(3-4) \times 10^{6}$ per SPS spill or $4.8 \mathrm{~s}$ produced by the primary $400 \mathrm{GeV} / \mathrm{c}$ proton beam with an intensity of a few $10^{12}$ protons on target. For study of the $\mu-\tau$ conversion it is planned to use the $150 \mathrm{GeV}$ muon beam from the M2 beamline with the muon intensity $\simeq 2 \times 10^{9}$ per SPS spill and intesity of $10^{13}$ protons on target.

\section{THEORETICAL SETUP}

We start with the low-energy effective Lagrangian relevant for two subprocesses of $e-\tau$ (ETC) and $\mu-\tau$ (MTC) conversion: (1) on quarks

$$
e^{-}+q_{i} \rightarrow \tau^{-}+q_{f}, \quad \mu^{-}+q_{i} \rightarrow \tau^{-}+q_{f}
$$

(2) and on antiquarks

$$
e^{-}+\bar{q}_{f} \rightarrow \tau^{-}+\bar{q}_{i}, \quad \mu^{-}+\bar{q}_{f} \rightarrow \tau^{-}+\bar{q}_{i} .
$$

Its most general form up to the dominant $\operatorname{dim}=6$ operators is

$\mathcal{L}_{\ell \tau}=\sum_{I, i f, X Y}\left(\Lambda_{I_{i f, X Y}}^{\ell \tau}\right)^{-2} \mathcal{O}_{I_{i f, X Y}^{\ell \tau}}^{\ell \tau}+$ H.c., $\quad \ell=e, \mu$,

where

$$
\begin{gathered}
\text { S-type: } \mathcal{O}_{S_{i f, X Y}^{\ell \tau}}^{\ell}=\left(\bar{\tau} P_{X} l\right)\left(\bar{q}_{f} P_{Y} q_{i}\right), \\
\text { V-type: } \mathcal{O}_{V_{i f, X Y}^{\ell \tau}}^{\ell \tau}=\left(\bar{\tau} \gamma^{\mu} P_{X} l\right)\left(\bar{q}_{f} \gamma_{\mu} P_{Y} q_{i}\right), \\
\mathbf{T} \text { - type: } \mathcal{O}_{T_{i f, X X}^{\ell \tau}}^{\ell \tau}=\left(\bar{\tau} \sigma^{\mu \nu} P_{X} l\right)\left(\bar{q}_{f} \sigma_{\mu \nu} P_{X} q_{i}\right)
\end{gathered}
$$

are $\operatorname{dim}=6$ operators with $\ell=e^{-}, \mu^{-}$. In Eq. (4), the summation over $I=S, V, T$, the quark flavors $i, f=u, d$, $s, c, b, t$ and chiralities $X, Y=L, R$ are implied. As usual, $P_{L, R}=\left(1 \mp \gamma_{5}\right) / 2$ are the chirality projection operators. The mass scales $\Lambda_{I_{i f, X Y}^{\ell \tau}}^{\ell \tau}$ set the strength of the low-energy effect of the corresponding operators. In total there are $360=6 \times 6 \times 10$ operators for the six quark flavors for each quark field and ten possible chirality combinations. These operators are subject to various already existing experimental constraints. Using these constraints we estimate the physics reach of the NA64 experiment [25] in the sense of the prospects of the observation of ETC and MTC (1) or improving the existing limits on the scales $\Lambda_{I_{i f, X Y}^{\ell} \tau}^{\ell}$. Prospects for the experimental searching for $\mu-\tau$ conversion in different context have been previously discussed in Refs. $[2,14,15]$ for the scalar operators.
Note that we use nonuniversal scales $\Lambda_{A B}^{\ell \tau}$ to characterize the strength of the corresponding low-energy effective pointlike operators. From the view point of a high-scale underlying theory the operators (5)-(7) represent lowenergy limits of the diagrams with two renormalizable vertices and a heavy intermediate particle of a typical mass $\mathcal{M}_{0}$. These diagrams are proportional to a product of two coupling constants, say, $g_{a}$ and $g_{b}$. We denote this product $\mathcal{C}_{A B}^{\ell \tau} \equiv g_{a} g_{b}$. After integrating out the heavy particles each operator goes accompanied with the factor

$$
\frac{\mathcal{C}_{A B}^{\ell \tau}}{M_{i}^{2}} \equiv \frac{1}{\left(\Lambda_{A B}^{\ell \tau}\right)^{2}}
$$

In what follows we derive lower limits on $\Lambda_{A B}^{\ell \tau}$. With the above relation one may easily translate our limits to limits on $M_{i}$ for certain values of the effective couplings $\mathcal{C}_{A B}^{\ell \tau}$. The latter depend on a high-scale model. For a weakly coupled high-scale model their "natural" values ${ }^{1}$ are

$$
\mathcal{C}_{A B}^{\ell \tau}=\mathcal{O}(1)
$$

Although the effective couplings can, in principle, be significantly smaller we use in our analysis-following the standard lore in the literature - their "natural" values (9) in order to assure the validity of effective low-energy description in terms of pointlike effective operators.

\section{OBSERVABLES}

First, we specify the kinematics. Let $P, p, p^{\prime}, k$, and $k^{\prime}$ be the momenta of initial nucleon, initial quark/final antiquark, final quark/initial antiquark, initial lepton, and final lepton, respectively. The set of invariant Mandelstam variables defining the kinematics of the quark/antiquark lepton scattering is given by

$$
\begin{aligned}
& \hat{s}=(k+p)^{2}=(k+x P)^{2}, \\
& \hat{t}=\left(k-k^{\prime}\right)^{2}, \\
& \hat{u}=\left(k-p^{\prime}\right)^{2},
\end{aligned}
$$

obeying the condition $\hat{s}+\hat{t}+\hat{u}=0$ for zero masses of quarks and nucleon in comparison with large value of initial lepton energy. Here $x$ is the Bjorken variable (the fraction of the nucleon momentum carried by $q_{i}$ or $\left.\bar{q}_{i}\right): x=Q^{2} /(q \cdot P)$. The inelasticity is $y=(q \cdot P) /(k \cdot P)$. The set $(\hat{s}, \hat{t}, \hat{u})$ is related to the total energy $s=(k+P)^{2} \simeq 2 m_{N} E_{\ell}$, where $m_{N}$ is the nucleon mass and $E_{\ell}$ is the lepton beam energy

$$
\begin{aligned}
& \hat{s}=s x, \\
& \hat{t}=q^{2}=-Q^{2}=-s x y, \\
& \hat{u}=-s x(1-y) .
\end{aligned}
$$

\footnotetext{
${ }^{1}$ For more details we refer reader to Ref. [15].
} 


\section{A. Integral cross section of the $\ell-\tau$ conversion}

Now using effective four-fermion operators we calculate the integral cross sections for the ETC and MTC. The total cross section of the $l-\tau$ conversion on a nucleus (1) can be approximated by the sum over the corresponding cross section on its constituent nucleons

$$
\begin{aligned}
\sigma(\ell+(A, Z) \rightarrow \tau+X)= & Z \sigma(\ell+p \rightarrow \tau+X) \\
& +(A-Z) \sigma(\ell+n \rightarrow \tau+X) .
\end{aligned}
$$

Here nucleon $N=p, n$ cross section is

$$
\begin{aligned}
& \sigma(\ell+N \rightarrow \tau+X) \\
& =\sum_{i f} \int_{0}^{1} d x \int_{0}^{1} d y\left[\frac{d^{2} \hat{\sigma}}{d x d y}\left(\ell+q_{i} \rightarrow \tau+q_{f}\right) q_{i}^{N}\left(x, Q^{2}\right)\right. \\
& \left.\quad+\frac{d^{2} \hat{\sigma}}{d x d y}\left(\ell+\bar{q}_{f} \rightarrow \tau+\bar{q}_{i}\right) \bar{q}_{f}^{N}\left(x, Q^{2}\right)\right],
\end{aligned}
$$

where $q_{i}^{N}\left(x, Q^{2}\right)$ and $\bar{q}_{i}^{N}\left(x, Q^{2}\right)$ are quark and antiquark PDFs, respectively. We will consider two nuclear targets: Fe with $A=56$ and $Z=26$ and $\mathrm{Pb}$ with $A=207$ and $Z=82$. Quark/antiquark PDFs depend on the resolution scale set by the square momentum transferred to the nucleon

$$
q^{2}=-Q^{2}=-\left(s-m_{N}^{2}-m_{l}^{2}\right) x y \simeq-s x y,
$$

where $m_{N}$ is the nucleon mass, $x=Q^{2} /(q \cdot P)$ is Bjorken variable, $y=(q \cdot P) /(k \cdot P)$ is inelasticity. Therefore, we should substitute $Q^{2}$ by sxy in Eq. (13). In the present paper we use quark PDFs from the CT10 next-to-next-to-leading order global analysis of QCD [26]. In fact, PDF fits using the standard CTEQ PDF evolution [27] but using the HOPPET $\alpha_{s}$ running solution.

The elementary differential cross sections corresponding to the contact 4-fermion interactions in Eq. (4) are given by

$$
\begin{aligned}
& \frac{d^{2} \hat{\sigma}}{d x d y}\left(\ell+q_{i} \rightarrow \tau+q_{f}\right)=\sum_{I, X Y} \frac{1}{\left(\Lambda_{I_{i f, X Y}}^{\ell \tau}\right)^{4}} \frac{\hat{s} f_{I, X Y}(y)}{64 \pi}, \\
& \frac{d^{2} \hat{\sigma}}{d x d y}\left(\ell+\bar{q}_{f} \rightarrow \tau+\bar{q}_{i}\right)=\sum_{I, X Y} \frac{1}{\left(\Lambda_{I_{i f, X Y}}^{\ell \tau}\right)^{4}} \frac{\hat{s} g_{I, X Y}(y)}{64 \pi} .
\end{aligned}
$$

Here $f_{I, X Y}(y)$ and $g_{I, X Y}(y)$ are functions related to the matrix elements of the effective operators (5)-(7). They are given in Appendix.

Substituting (15), (16) into (13) and (12) we find

$$
\sigma(\ell+(A, Z) \rightarrow \tau+X)=\sum_{I, i f, X Y} \frac{Q_{I_{i f, X Y}^{A}}^{A}}{\Lambda_{I_{i f, X Y}}^{4}}
$$

with

$$
\begin{aligned}
Q_{I_{i f, X Y}^{A}}^{A}= & \frac{s}{64 \pi} \int_{0}^{1} d x \int_{0}^{1} d y\left[x f_{I, X Y}(y) q_{i}^{A}(x, s x y)\right. \\
& \left.+x g_{I, X Y}(y) \bar{q}_{f}^{A}(x, s x y)\right],
\end{aligned}
$$

where

$$
\begin{aligned}
u^{A}\left(x, Q^{2}\right) & =Z u^{p}\left(x, Q^{2}\right)+(A-Z) d^{p}\left(x, Q^{2}\right), \\
d^{A}\left(x, Q^{2}\right) & =Z d^{p}\left(x, Q^{2}\right)+(A-Z) u^{p}\left(x, Q^{2}\right), \\
u^{A}\left(x, Q^{2}\right)+d^{A}\left(x, Q^{2}\right) & =A\left(u^{p}\left(x, Q^{2}\right)+d^{p}\left(x, Q^{2}\right)\right), \\
\bar{u}^{A}\left(x, Q^{2}\right) & =A \bar{u}^{p}\left(x, Q^{2}\right), \\
\bar{d}^{A}\left(x, Q^{2}\right) & =A \bar{d}^{p}\left(x, Q^{2}\right), \\
s^{A}\left(x, Q^{2}\right)=\bar{s}^{A}\left(x, Q^{2}\right) & =A s^{p}\left(x, Q^{2}\right), \\
c^{A}\left(x, Q^{2}\right)=\bar{c}^{A}\left(x, Q^{2}\right) & =A c^{p}\left(x, Q^{2}\right), \\
b^{A}\left(x, Q^{2}\right)=\bar{b}^{A}\left(x, Q^{2}\right) & =A b^{p}\left(x, Q^{2}\right)
\end{aligned}
$$

\begin{tabular}{|c|c|c|c|}
\hline$(\operatorname{IiXY})$ & $Q_{I_{i f, X Y}}^{A}$ & $(I i X Y)$ & $Q_{I_{i f, X Y}}^{A}$ \\
\hline \multicolumn{4}{|c|}{$S$ operators } \\
\hline$(\mathrm{SuXY})$ & 3.82 & $(S d X Y)$ & 4.07 \\
\hline$\left(S_{s} X Y\right)$ & 0.74 & $(S c X Y)$ & 0.21 \\
\hline$(\operatorname{SbXY})$ & 0.006 & & \\
\hline \multicolumn{4}{|c|}{$V$ operators } \\
\hline$(V u L L / R R)$ & 43.83 & $(V u L R / R L)$ & 20.51 \\
\hline$(V d L L / R R)$ & 46.23 & $(V d L R / R L)$ & 22.46 \\
\hline$(V s L L / R R)$ & 5.85 & $(V s L R / R L)$ & 5.85 \\
\hline$(V c L L / R R)$ & 1.41 & $(V c L R / R L)$ & 1.41 \\
\hline$(V b L L / R R)$ & 0.02 & $(V b L R / R L)$ & 0.02 \\
\hline \multicolumn{4}{|c|}{$T$ operators } \\
\hline$(T u L L / R R)$ & 453.52 & $(T d L L / R R)$ & 484.37 \\
\hline$(T s L L / R R)$ & 81.84 & $(T c L L / R R)$ & 19.21 \\
\hline$(T b L L / R R)$ & 0.23 & & \\
\hline
\end{tabular}

are the quark and antiquark PDFs in a nucleus $A$. Numerical results for the double moments $Q_{I_{i f, X Y}}^{A}$ are shown in Tables I-IV for $\mathrm{Fe}$ and $\mathrm{Pb}$ nuclear targets and for the electron and muon beams.

The dominant contribution to the inclusive $\ell+A$ cross section is due to the bremsstrahlung of leptons on nuclei, given by the formula $[28,29]$

$\sigma_{B S}(\ell+(A, Z) \rightarrow \ell+X)=4 \alpha r_{\ell}^{2} Z^{2}\left[\frac{7}{9} \log \left(\frac{183}{Z^{1 / 3}} \frac{m_{\ell}}{m_{e}}\right)\right]$

TABLE I. Double moments of quark PDF $Q_{I_{i f X Y}^{A}}^{A}$ (in $\mathrm{GeV}^{2}$ ) with $f=u, d, s, c, b$ and $i$ specified in the Table. The case of a Fe target and an electron beam with $E_{e}=100 \mathrm{GeV}$. 
TABLE II. The same as in Table I, but for a muon beam with $E_{\mu}=150 \mathrm{GeV}$.

\begin{tabular}{|c|c|c|c|}
\hline$(I i X Y)$ & $Q_{I_{i f, X Y}}^{A}$ & $(I i X Y)$ & $Q_{I_{i f, X Y}^{A}}^{A}$ \\
\hline \multicolumn{4}{|c|}{$S$ operators } \\
\hline$(S u X Y)$ & 5.64 & $(S d X Y)$ & 6.01 \\
\hline$(S s X Y)$ & 1.12 & $(S c X Y)$ & 0.35 \\
\hline$(S b X Y)$ & 0.02 & & \\
\hline \multicolumn{4}{|c|}{$V$ operators } \\
\hline$(V u L L / R R)$ & 64.30 & $(V u L R / R L)$ & 30.21 \\
\hline$(V d L L / R R)$ & 67.81 & $(V d L R / R L)$ & 33.07 \\
\hline$\left(V_{s} L L / R R\right)$ & 8.84 & $(V s L R / R L)$ & 8.84 \\
\hline$(V c L L / R R)$ & 2.32 & $(V c L R / R L)$ & 2.32 \\
\hline$(V b L L / R R)$ & 0.07 & $(V b L R / R L)$ & 0.07 \\
\hline \multicolumn{4}{|c|}{$T$ operators } \\
\hline$(T u L L / R R)$ & 665.88 & $(T d L L / R R)$ & 710.69 \\
\hline$(T s L L / R R)$ & 123.48 & $(T c L L / R R)$ & 31.57 \\
\hline$(T b L L / R R)$ & 0.79 & & \\
\hline
\end{tabular}

where $r_{\ell}=e^{2} /\left(4 \pi \epsilon_{0} m_{\ell} c^{2}\right)$ is the classical lepton radius: $2.818 \mathrm{fm}$ (for $e$ ) and $0.0136 \mathrm{fm}$ (for $\mu$ ). For specific beam and target we have numerically

$$
\begin{aligned}
& \sigma_{B S}(e+\mathrm{Fe} \rightarrow e+X)=0.129 \times 10^{5} \mathrm{GeV}^{-2}, \\
& \sigma_{B S}(e+\mathrm{Pb} \rightarrow e+X)=1.165 \times 10^{5} \mathrm{GeV}^{-2}, \\
& \sigma_{B S}(\mu+\mathrm{Fe} \rightarrow \mu+X)=0.692 \mathrm{GeV}^{-2}, \\
& \sigma_{B S}(\mu+\mathrm{Pb} \rightarrow \mu+X)=6.607 \mathrm{GeV}^{-2},
\end{aligned}
$$

which will be used in the following section for the extraction of the limits on $\mu(e)-\tau \mathrm{LFV}$ form the expected sensitivity of the NA64 experiment.

TABLE III. The same as in Table I, but for a $\mathrm{Pb}$ and an electron beam with $E_{e}=100 \mathrm{GeV}$.

\begin{tabular}{|c|c|c|c|}
\hline$(I i X Y)$ & $Q_{I_{i f, X Y}^{A}}^{A}$ & $(I i X Y)$ & $Q_{I_{i f, X Y}}^{A}$ \\
\hline \multicolumn{4}{|c|}{$S$ operators } \\
\hline$(S u X Y)$ & 20.03 & $(S d X Y)$ & 23.02 \\
\hline$(S s X Y)$ & 4.14 & $(S c X Y)$ & 1.28 \\
\hline$(S b X Y)$ & 0.07 & & \\
\hline \multicolumn{4}{|c|}{$V$ operators } \\
\hline$(V u L L / R R)$ & 227.61 & $(V u L R / R L)$ & 108.12 \\
\hline$(V d L L / R R)$ & 260.74 & $(V d L R / R L)$ & 125.80 \\
\hline$(V s L L / R R)$ & 32.67 & $(V S L R / R L)$ & 32.67 \\
\hline$(V c L L / R R)$ & 8.57 & $(V c L R / R L)$ & 8.57 \\
\hline$(V b L L / R R)$ & 0.25 & $(V b L R / R L)$ & 0.25 \\
\hline \multicolumn{4}{|c|}{$T$ operators } \\
\hline$(T u L L / R R)$ & 2365.21 & $(T d L L / R R)$ & 2723.18 \\
\hline$(T s L L / R R)$ & 456.45 & $(T c L L / R R)$ & 116.71 \\
\hline$(T b L L / R R)$ & 2.92 & & \\
\hline
\end{tabular}

\begin{tabular}{lrrr}
\hline \hline$(I i X Y)$ & $Q_{I_{i f, X Y}}^{A}$ & $(I i X Y)$ & $Q_{I_{i f, X Y}}^{A}$ \\
\hline \multicolumn{4}{c}{$S$ operators } \\
$(S u X Y)$ & 13.58 & $(S d X Y)$ & 15.61 \\
$(S s X Y)$ & 2.73 & $(S c X Y)$ & 0.77 \\
$(S b X Y)$ & 0.02 & & \\
\multicolumn{4}{c}{} \\
$(V u L L / R R)$ & 155.11 & $(V u L R / R L)$ & 73.38 \\
$(V d L L / R R)$ & 177.78 & $(V d L R / R L)$ & 85.47 \\
$(V s L L / R R)$ & 21.63 & $(V s L R / R L)$ & 21.63 \\
$(V c L L / R R)$ & 5.20 & $(V c L R / R L)$ & 5.20 \\
$(V b L L / R R)$ & 0.08 & $(V b L R / R L)$ & 0.08 \\
\multicolumn{5}{c}{ operators } \\
$(T u L L / R R)$ & 1610.52 & $(T d L L / R R)$ & 1856.30 \\
$(T s L L / R R)$ & 302.50 & $(T c L L / R R)$ & 71.00 \\
$(T b L L / R R)$ & 0.85 & & \\
\hline \hline
\end{tabular}

TABLE IV. The same as in Table III, but for a muon beam with $E_{\mu}=150 \mathrm{GeV}$.

\section{B. Energy Spectrum}

An important characteristic helping to plan the $\ell-\tau$ conversion experiments is the energy spectrum of the final $\tau$-lepton defined as

$$
\begin{aligned}
\mathcal{F}_{I}\left(E_{\ell}, E_{\tau}\right)= & \frac{1}{\sigma_{I}(l+(A, Z) \rightarrow \tau+X)} \\
& \times \frac{d \sigma_{I}(l+(A, Z) \rightarrow \tau+X)}{d E_{\tau}},
\end{aligned}
$$

where $\sigma_{I}$ is the total cross section assuming the single operator $I=S, V, T$ dominance and the differential cross section is given by

$$
\begin{aligned}
\frac{d \sigma_{I}}{d E_{\tau}}= & \sum_{i f, X Y} \frac{M_{N}}{32 \pi \Lambda_{I_{i f, X Y}}^{4}} \int_{0}^{1} d x\left[x q_{i}^{A}\left(x, \mu^{2}\right) f_{I, X Y}(1-z)\right. \\
& \left.+x \bar{q}_{f}^{A}\left(x, \mu^{2}\right) g_{I, X Y}(1-z)\right]
\end{aligned}
$$

with $\mu^{2}=s x(1-z)$ and $z=E_{\tau} / E_{\ell}$ running from 0 to 1 , which corresponds to $0 \leq E_{\tau} \leq E_{\ell}=s /\left(2 M_{N}\right)$. Here we use $d \hat{\sigma} / d E_{\tau}=-\left(2 M_{N} / s\right)(d \hat{\sigma} / d z)$. Note, the quantity $\mathcal{F}_{I}\left(E_{\ell}, E_{\tau}\right)$ is independent of the LFV scales $\Lambda_{I}$. It is also independent of the target nucleus, since we sum over all the initial quark flavors $i$. In Figs. 1-3 we plot the energy spectra $\mathcal{F}_{I}\left(E_{\ell}, E_{\tau}\right)$ for $I=S, V, T$ disregarding the quark contributions subdominant in comparison with $u+d$ quark and antiquark contribution. For simplicity, for each type of the LFV operator with specific spin structure $I=S, V, T$, we suppose the same value of the coupling $\Lambda_{I}$ independent of the quark flavor. We use the following notations: $e_{F}$ and $\mu_{F}$ are the full contributions (including all species of quark and antiquarks) in case of the $E_{e}=100 \mathrm{GeV}$ electron and $E_{\mu}=150 \mathrm{GeV}$ muon beam, respectively; $e_{u d}$ and $\mu_{u d}$ are 


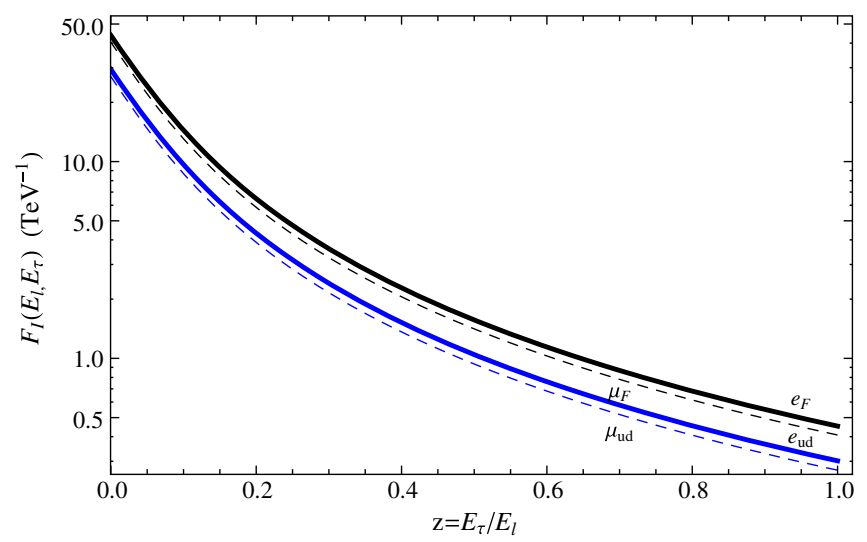

FIG. 1. Spectrum of $\tau$ lepton for $S$ operators.

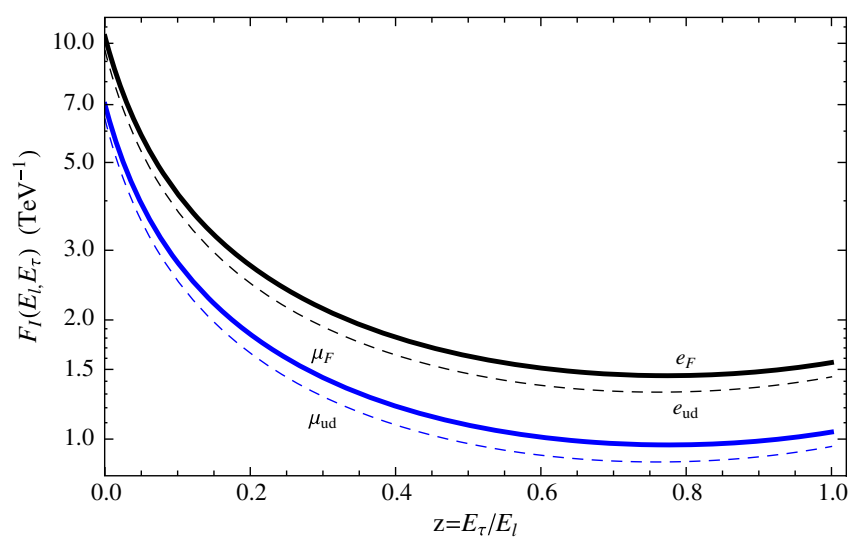

FIG. 2. Spectrum of $\tau$ lepton for $V$ operators.

the respective $u+d$ contributions for the same values of energies of $e$ and $\mu$ beam.

A useful "integral" quantity is the mean energy $\left\langle E_{\tau}\right\rangle$ of the final $\tau$ lepton defined as

$\left\langle E_{\tau}\right\rangle_{I}=\int_{0}^{E_{\ell}} d E_{\tau} E_{\tau} \mathcal{F}_{I}\left(E_{\ell}, E_{\tau}\right) \equiv E_{\ell} \frac{\sum_{i f, X Y} \tilde{Q}_{I_{i f, X Y}^{A}}^{A}}{\sum_{i f, X Y} Q_{I_{i f, X Y}}^{A}}$,

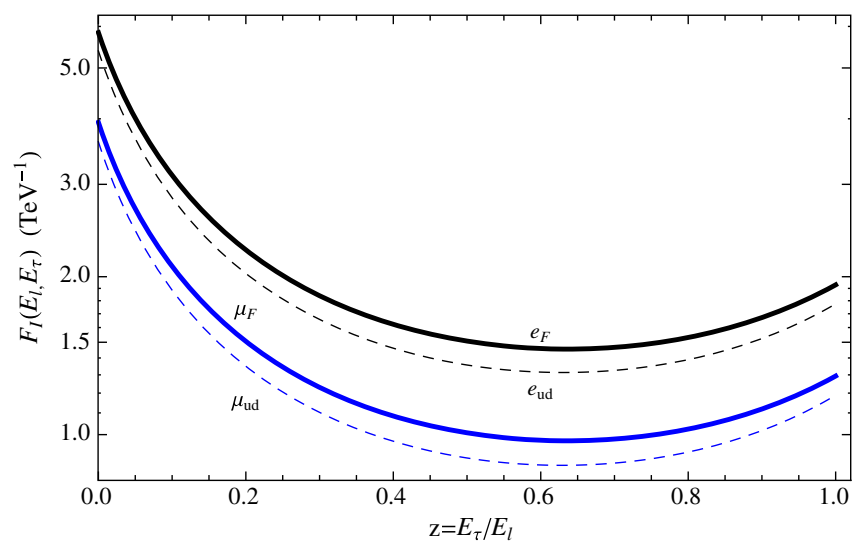

FIG. 3. Spectrum of $\tau$ lepton for $T$ operators.
TABLE V. Double moments of quark PDF $\tilde{Q}_{I_{i f . X Y}}^{A}$ (in $\mathrm{GeV}^{2}$ ) with $f=u, d, s, c, b$ and $i$ specified in the Table. The case of a Fe target and an electron beam with $E_{e}=100 \mathrm{GeV}$.

\begin{tabular}{|c|c|c|c|}
\hline$(\operatorname{IiXY})$ & $\tilde{Q}_{I_{i f, X Y}}^{A}$ & $(I i X Y)$ & $\tilde{Q}_{I_{i f, X Y}}^{A}$ \\
\hline \multicolumn{4}{|c|}{$S$ operators } \\
\hline$(S u X Y)$ & 0.97 & $(S d X Y)$ & 1.03 \\
\hline$(S s X Y)$ & 0.18 & $(S c X Y)$ & 0.05 \\
\hline$(S b X Y)$ & 0.001 & & \\
\hline \multicolumn{4}{|c|}{$V$ operators } \\
\hline$(V u L L / R R)$ & 23.08 & $(V u L R / R L)$ & 14.12 \\
\hline$(V d L L / R R)$ & 24.43 & $(V d L R / R L)$ & 15.31 \\
\hline$(V s L L / R R)$ & 3.28 & $(V s L R / R L)$ & 3.28 \\
\hline$(V c L L / R R)$ & 0.72 & $(V c L R / R L)$ & 0.72 \\
\hline$(V b L L / R R)$ & 0.005 & $(V b L R / R L)$ & 0.005 \\
\hline \multicolumn{4}{|c|}{$T$ operators } \\
\hline$(T u L L / R R)$ & 282.06 & $(T d L L / R R)$ & 301.39 \\
\hline$(T s L L / R R)$ & 49.55 & $(T c L L / R R)$ & 10.79 \\
\hline$(T b L L / R R)$ & 0.06 & & \\
\hline
\end{tabular}

where

$$
\begin{aligned}
\tilde{Q}_{I_{i f, X Y}^{A}}^{A}= & \frac{s}{64 \pi} \int_{0}^{1} d x x \int_{0}^{1} d z z\left[q_{i}^{A}(x, s x(1-z)) f_{I, X Y}(1-z)\right. \\
& \left.+\bar{q}_{f}^{A}(x, s x(1-z)) g_{I, X Y}(1-z)\right]
\end{aligned}
$$

separately for each of the operators $I=S, V, T$ in Eqs. (5)-(7). Here, as in case of Figs. 1-3 for each type of the LFV operator with specific spin structure $I=S, V, T$ we use the same value of the coupling $\Lambda_{I}$ independent of the quark flavor. Note, that $E_{\tau}$ is independent of type of nucleus target because double moments of quark/antiquark $\tilde{Q}_{I_{i f, X Y}^{A}}^{A}$ and $Q_{I_{i f, X Y}^{A}}^{A}$ are both proportional to $A$.

For the definition of $Q^{A}$ see Eq. (18). Our predictions for $\tilde{Q}_{I_{i f, X Y}^{A}}^{A}$ and $\left\langle E_{\tau}\right\rangle_{I}$ are displayed in Tables V-VIII and in

TABLE VI. The same as in Table V, but for a muon beam with

\begin{tabular}{|c|c|c|c|}
\hline$(I i X Y)$ & $\tilde{Q}_{I_{i f, X Y}}^{A}$ & $(I i X Y)$ & $\tilde{Q}_{I_{i f, X Y}}^{A}$ \\
\hline \multicolumn{4}{|c|}{$S$ operators } \\
\hline$(S u X Y)$ & 1.42 & $(S d X Y)$ & 1.52 \\
\hline$(S s X Y)$ & 0.28 & $(S c X Y)$ & 0.08 \\
\hline$(S b X Y)$ & 0.003 & & \\
\hline \multicolumn{4}{|c|}{$V$ operators } \\
\hline$(V u L L / R R)$ & 33.82 & $(V u L R / R L)$ & 20.75 \\
\hline$(V d L L / R R)$ & 35.79 & $(V d L R / R L)$ & 22.48 \\
\hline$(V s L L / R R)$ & 4.94 & $(V s L R / R L)$ & 4.94 \\
\hline$(V c L L / R R)$ & 1.18 & $(V c L R / R L)$ & 1.18 \\
\hline$(V b L L / R R)$ & 0.02 & $(V b L R / R L)$ & 0.02 \\
\hline \multicolumn{4}{|c|}{$T$ operators } \\
\hline$(T u L L / R R)$ & 413.67 & $(T d L L / R R)$ & 441.89 \\
\hline$(T s L L / R R)$ & 74.58 & $(T c L L / R R)$ & 17.53 \\
\hline$(T b L L / R R)$ & 0.24 & & \\
\hline
\end{tabular}
$E_{\mu}=150 \mathrm{GeV}$. 
TABLE VII. The same as in Table $\mathrm{V}$, but for a $\mathrm{Pb}$ target and an electron beam with $E_{e}=100 \mathrm{GeV}$.

\begin{tabular}{|c|c|c|c|}
\hline$(\operatorname{IiXY})$ & $\tilde{Q}_{I_{i f, X Y}}^{A}$ & $(I i X Y)$ & $\tilde{Q}_{I_{i f, X Y}^{A}}^{A}$ \\
\hline \multicolumn{4}{|c|}{$S$ operators } \\
\hline$(S u X Y)$ & 3.44 & $(S d X Y)$ & 3.96 \\
\hline$\left(S_{S} X Y\right)$ & 0.68 & $(S c X Y)$ & 0.18 \\
\hline$(S b X Y)$ & 0.004 & & \\
\hline \multicolumn{4}{|c|}{$V$ operators } \\
\hline$(V u L L / R R)$ & 81.73 & $(V u L R / R L)$ & 50.33 \\
\hline$(V d L L / R R)$ & 93.87 & $(V d L R / R L)$ & 58.45 \\
\hline$(V s L L / R R)$ & 12.13 & $(V s L R / R L)$ & 12.13 \\
\hline$(V c L L / R R)$ & 2.67 & $(V c L R / R L)$ & 2.67 \\
\hline$(V b L L / R R)$ & 0.02 & $(V b L R / R L)$ & 0.02 \\
\hline \multicolumn{4}{|c|}{$T$ operators } \\
\hline$(T u L L / R R)$ & 1001.40 & $(T d L L / R R)$ & 1155.30 \\
\hline$(T s L L / R R)$ & 183.18 & $(T c L L / R R)$ & 39.88 \\
\hline$(T b L L / R R)$ & 0.22 & & \\
\hline
\end{tabular}

TABLE VIII. The same as in Table VII, but for a muon beam with $E_{\mu}=150 \mathrm{GeV}$.

\begin{tabular}{|c|c|c|c|}
\hline$(\operatorname{IiXY})$ & $\tilde{Q}_{I_{i f, X Y}^{A}}^{A}$ & $(I i X Y)$ & $\tilde{Q}_{I_{i f, X Y}}^{A}$ \\
\hline \multicolumn{4}{|c|}{$S$ operators } \\
\hline$(S u X Y)$ & 5.06 & $(S d X Y)$ & 5.82 \\
\hline$(S s X Y)$ & 1.03 & $(S c X Y)$ & 0.29 \\
\hline$(S b X Y)$ & 0.02 & & \\
\hline \multicolumn{4}{|c|}{$V$ operators } \\
\hline$(V u L L / R R)$ & 119.80 & $(V u L R / R L)$ & 74.00 \\
\hline$(V d L L / R R)$ & 137.52 & $(V d L R / R L)$ & 85.79 \\
\hline$(V s L L / R R)$ & 18.26 & $(V s L R / R L)$ & 18.26 \\
\hline$(V c L L / R R)$ & 4.35 & $(V c L R / R L)$ & 4.35 \\
\hline$(V b L L / R R)$ & 0.07 & $(V b L R / R L)$ & 0.07 \\
\hline \multicolumn{4}{|c|}{$T$ operators } \\
\hline$(T u L L / R R)$ & 1469.25 & $(T d L L / R R)$ & 1693.52 \\
\hline$(T s L L / R R)$ & 275.69 & $(T c L L / R R)$ & 64.82 \\
\hline$(T b L L / R R)$ & 0.89 & & \\
\hline
\end{tabular}

Tables IX and $\mathrm{X}$, respectively, for $\ell=e, \mu$ beams and $\mathrm{Fe}$, $\mathrm{Pb}$ targets.

For experiments searching for the LFV process (1), it is crucial that the missing energy in the decay of the final $\tau$-lepton be above some value. This is needed for the suppression of the typical backgrounds. For the NA64

TABLE IX. $\left\langle E_{\tau}\right\rangle_{I}$ (in GeV) for different LFV operators in the case of an electron beam with $E_{e}=100 \mathrm{GeV}$.

\begin{tabular}{lc}
\hline \hline Operator & $\left\langle E_{\tau}\right\rangle_{I}$ \\
\hline$S$ operators & 25 \\
$V$ operators & 57 \\
$T$ operators & 62 \\
\hline \hline
\end{tabular}

TABLE X. The same as in Table IX, but for a muon beam with $E_{\mu}=150 \mathrm{GeV}$.

\begin{tabular}{lc}
\hline \hline Operator & $\left\langle E_{\tau}\right\rangle_{I}$ \\
\hline$S$ operators & 38 \\
$V$ operators & 86 \\
$T$ operators & 93 \\
\hline \hline
\end{tabular}

experiment, this cutoff is preliminarily estimated to be in the range $10-30 \mathrm{GeV}$ (the detailed simulation results will be reported elsewhere). Thus, the $E_{\tau}$ should be large than the value. For example, for the cutoff of $10 \mathrm{GeV}$, the mean energy $\left\langle E_{\tau}\right\rangle_{I}$ of the final $\tau$-lepton is significantly larger than this value for the contribution of all the operators (5)-(7) as one can seen from Tables IX and X.

\section{LIMITS ON THE LFV SCALES}

Here we derive limits on the mass scales $\Lambda$ of the LFV operators in Eqs. (5)-(7) and compare them with the corresponding limits existing in the literature.

\section{A. Expected limits from NA64 experiment}

The quantity of the interest in the planning measurements of the electron (muon)-tau lepton conversion is the ratio:

$$
R_{\ell \tau}=\frac{\sigma(\ell+A \rightarrow \tau+X)}{\sigma(\ell+A \rightarrow \ell+X)}
$$

where $\sigma(\ell+A \rightarrow \ell+X) \approx \sigma_{B S}(\ell+A \rightarrow \ell+X)$.

The physics reach of the NA64 experiment in this quantity is expected to be at the level of

$$
R_{\ell \tau} \sim 10^{-13}-10^{-12}
$$

Assuming single operator dominance, the most optimistic value of (27) would result in the constraints on the LFV scales $\Lambda_{I_{i f, X Y}}$ shown in Tables XI-XIV. As seen from these tables, the limits are in the ranges

$$
\begin{aligned}
& S-\text { operators: } \Lambda^{e \tau} \geq 0.04-0.24 \mathrm{TeV}, \\
& \Lambda^{\mu \tau} \geq 0.56-3.05 \mathrm{TeV}, \\
& V-\text { operators: } \Lambda^{e \tau} \geq 0.05-0.44 \mathrm{TeV}, \\
& \Lambda^{\mu \tau} \geq 0.78-5.60 \mathrm{TeV}, \\
& T-\text { operators: } \Lambda^{e \tau} \geq 0.09-0.66 \mathrm{TeV}, \\
& \Lambda^{\mu \tau} \geq 1.45-10.06 \mathrm{TeV} .
\end{aligned}
$$

The worst limits are set on the operators with b-quark. Such low scales may look incompatible with the concept of effective low-energy pointlike 4-fermion interactions (4)-(7) used in our analysis. In this respect the following 
comments are in order. First of all, the low values for the lower bounds of $\Lambda_{A B}^{\ell \tau}$ indicate deficiency of the method of their derivation saying nothing about their actual values. It is believed that the scales of the LFV operators are at a scale of $\gtrsim 1 \mathrm{TeV}$. Alas, for the weakly constrained scales, shown in (28), there are no experimental constraints as yet. Also, as we pointed out at the end of Sec. II, we assume natural values (9) of the effective couplings of a high-scale model underlying the effective low-energy Lagrangian (4). Then, for the masses of heavy LFV mediators we have $M_{0} \sim \Lambda_{A B}^{\ell \tau}$. Deviation from the pointlike regime of the interactions (4) would manifest itself in the propagator effect

$$
\frac{1}{M_{0}^{2}} \rightarrow \frac{1}{\hat{t}+M_{0}^{2}}
$$

for the particle exchange in the $t$-channel and analogously in $u, s-$ channels. From Eqs. (11) it follows for the average values $\langle\hat{t}\rangle,\langle\hat{s}\rangle,\langle\hat{u}\rangle \leq 94(140) \mathrm{GeV}^{2}$ for the electron $E_{e}=$ $100 \mathrm{GeV}$ (muon $E_{\mu}=150 \mathrm{GeV}$ ) beam energy at NA64, which, for $M_{0}^{2} \geq 1600 \mathrm{GeV}^{2}$, is going to result in a mild propagator effect irrelevant for our rough estimations. An exception may happen for a leptoquark (LQ) with the mass $M_{L Q}^{2} \leq 2 m_{N} E_{\ell}=200(300) \mathrm{GeV}^{2}$ in the $s$-channel for $E_{e}=100 \mathrm{GeV}\left(E_{\mu}=150 \mathrm{GeV}\right)$. In this case, the pointlike picture breaks down and the on-shell LQ is produced in $\ell-q$-collision. However, the value $M_{L Q} \lesssim 20 \mathrm{GeV}$ even for an LQ coupled only to $b-e$, for which there are neither direct nor indirect constraints, such a small value looks unlikely.

Let us note that our limits shown in Tables XI-XIV have been derived from the best expected sensitivity (27) of the NA64 experiment and may look too optimistic. However,

TABLE XI. Lower limits for LFV mass scales $\Lambda_{I_{i f, X Y}}$ (in TeV) of the operators in Eq. (4) with $f=u, d, s, c, b$ and $i$ specified in the Table. The case of a Fe target and an electron beam with $E_{e}=100 \mathrm{GeV}$.

\begin{tabular}{|c|c|c|c|}
\hline$(I i X Y)$ & $\Lambda_{I_{i f, X Y}}$ & $(I i X Y)$ & $\Lambda_{I_{i f, X Y}}$ \\
\hline \multicolumn{4}{|c|}{$S$ operators } \\
\hline$(S u X Y)$ & 0.23 & $(S d X Y)$ & 0.24 \\
\hline$(S s X Y)$ & 0.15 & $(S c X Y)$ & 0.11 \\
\hline$(S b X Y)$ & 0.05 & & \\
\hline \multicolumn{4}{|c|}{$V$ operators } \\
\hline$(V u L L / R R)$ & 0.43 & $(V u L R / R L)$ & 0.35 \\
\hline$(V d L L / R R)$ & 0.44 & $(V d L R / R L)$ & 0.36 \\
\hline$(V s L L / R R)$ & 0.26 & $(V s L R / R L)$ & 0.26 \\
\hline$(V c L L / R R)$ & 0.18 & $(V c L R / R L)$ & 0.18 \\
\hline$(V b L L / R R)$ & 0.06 & $(V b L R / R L)$ & 0.06 \\
\hline \multicolumn{4}{|c|}{$T$ operators } \\
\hline$(T u L L / R R)$ & 0.77 & $(T d L L / R R)$ & 0.78 \\
\hline$(T s L L / R R)$ & 0.50 & $(T c L L / R R)$ & 0.34 \\
\hline$(T b L L / R R)$ & 0.12 & & \\
\hline
\end{tabular}

TABLE XII. The same as in Table XI, but for a muon beam with $E_{\mu}=150 \mathrm{GeV}$.

\begin{tabular}{|c|c|c|c|}
\hline$(I i X Y)$ & $\Lambda_{I_{i f, X Y}}$ & $(I i X Y)$ & $\Lambda_{I_{i f, X Y}}$ \\
\hline \multicolumn{4}{|c|}{$S$ operators } \\
\hline$(S u X Y)$ & 3.00 & $(S d X Y)$ & 3.05 \\
\hline$(S s X Y)$ & 2.01 & $(S c X Y)$ & 1.50 \\
\hline$(S b X Y)$ & 0.72 & & \\
\hline \multicolumn{4}{|c|}{$V$ operators } \\
\hline$(V u L L / R R)$ & 5.52 & $(V u L R / R L)$ & 4.57 \\
\hline$(V d L L / R R)$ & 5.60 & $(V d L R / R L)$ & 4.67 \\
\hline$(V s L L / R R)$ & 3.36 & $(V s L R / R L)$ & 3.36 \\
\hline$(V c L L / R R)$ & 2.41 & $(V c L R / R L)$ & 2.41 \\
\hline$(V b L L / R R)$ & 1.00 & $(V b L R / R L)$ & 1.00 \\
\hline \multicolumn{4}{|c|}{$T$ operators } \\
\hline$(T u L L / R R)$ & 9.90 & $(T d L L / R R)$ & 10.06 \\
\hline$(T s L L / R R)$ & 6.50 & $(T c L L / R R)$ & 4.62 \\
\hline$(T b L L / R R)$ & 1.84 & & \\
\hline
\end{tabular}

lower limits on $\Lambda$ 's extracted from an experimental upper bound on $R_{l \tau}$ scale as $\left(R_{l \tau}\right)^{1 / 4}$ and, therefore, for the less optimistic case of $R_{\ell \tau} \sim 10^{-12}$, or even worse, they will be comparable with the limits in our Tables and still valuable as they are obtained for the first time.

\section{B. Limits from other experiments}

In the literature, there exist limits on many of the operators (5)-(7). As a reference point, from the accelerator experiments let us mention the constraint from the ZEUS experiment [1] at HERA (DESY), which is

$$
\Lambda_{\text {ZEUS }}^{e \tau} \geq 0.41-1.86 \mathrm{TeV}
$$

TABLE XIII. The same as in Table XI, but for a $\mathrm{Pb}$ target and an electron beam with $E_{e}=100 \mathrm{GeV}$.

\begin{tabular}{llcc}
\hline \hline$(I i X Y)$ & $\Lambda_{I_{i f, X Y}}$ & $(\operatorname{IiXY})$ & $\Lambda_{I_{i f, X Y}}$ \\
\hline \multicolumn{4}{c}{$S$ operators } \\
$(S u X Y)$ & 0.18 & $(S d X Y)$ & 0.19 \\
$(S s X Y)$ & 0.12 & $(S c X Y)$ & 0.09 \\
$(S b X Y)$ & 0.04 & & \\
\multicolumn{4}{r}{} \\
$(V u L L / R R)$ & 0.34 & $(V u L R / R L)$ & 0.28 \\
$(V d L L / R R)$ & 0.35 & $(V d L R / R L)$ & 0.29 \\
$(V s L L / R R)$ & 0.21 & $(V s L R / R L)$ & 0.21 \\
$(V c L L / R R)$ & 0.15 & $(V c L R / R L)$ & 0.15 \\
$(V b L L / R R)$ & 0.05 & $(V b L R / R L)$ & 0.05 \\
& \multicolumn{4}{c}{$T$ operators } \\
$(T u L L / R R)$ & 0.61 & $(T d L L / R R)$ & 0.63 \\
$(T s L L / R R)$ & 0.40 & $(T c L L / R R)$ & 0.28 \\
$(T b L L / R R)$ & 0.09 & & \\
\hline \hline
\end{tabular}


TABLE XIV. The same as in Table XIII, but for a muon beam with $E_{\mu}=150 \mathrm{GeV}$.

\begin{tabular}{|c|c|c|c|}
\hline$(\operatorname{IiXY})$ & $\Lambda_{I_{i f, X Y}}$ & $(I i X Y)$ & $\Lambda_{I_{i f, X Y}}$ \\
\hline \multicolumn{4}{|c|}{$S$ operators } \\
\hline$(S u X Y)$ & 2.35 & $(S d X Y)$ & 2.45 \\
\hline$(S s X Y)$ & 1.58 & $(S c X Y)$ & 1.17 \\
\hline$(S b X Y)$ & 0.56 & & \\
\hline \multicolumn{4}{|c|}{$V$ operators } \\
\hline$(V u L L / R R)$ & 4.31 & $(V u L R / R L)$ & 3.57 \\
\hline$(V d L L / R R)$ & 4.46 & $(V d L R / R L)$ & 3.71 \\
\hline$(V s L L / R R)$ & 2.65 & $(V s L R / R L)$ & 2.65 \\
\hline$(V c L L / R R)$ & 1.90 & $(V c L R / R L)$ & 1.90 \\
\hline$(V b L L / R R)$ & 0.78 & $(V b L R / R L)$ & 0.78 \\
\hline \multicolumn{4}{|c|}{$T$ operators } \\
\hline$(T u L L / R R)$ & 7.74 & $(T d L L / R R)$ & 8.01 \\
\hline$(T s L L / R R)$ & 5.13 & $(T c L L / R R)$ & 3.65 \\
\hline$(T b L L / R R)$ & 1.45 & & \\
\hline
\end{tabular}

These limits apply to the scales for all the operators in Eqs. (5)-(7), but only for the first generation quarks $u, d$. Note that Ref. [1] used another basis of the four-fermion operators motivated by the leptoquark exchange, which we adjusted to our in Eqs. (5)-(7) and thus obtained (30) from the limits on the leptoquark mass in Ref. [1].

Limits on the operators (5)-(7) can be extracted from the decays,

$$
\begin{aligned}
\tau & \rightarrow \ell+M^{0}, \\
B & \rightarrow \ell+\tau, \\
B & \rightarrow \ell^{ \pm}+\tau^{\mp}+M,
\end{aligned}
$$

where $M$ is a generic meson allowed by energy-momentum conservation. Using the existing experimental bounds in Ref. [15], the authors extracted limits from some of these processes on the scales of the scalar, pseudoscalar, vector and axial-vector $\mu-\tau$ LFV effective operators, which differ from our basis (5)-(7). Thus, their limits translate to limits on linear combinations of our operators. Assuming no significant cancelations in these combinations we have the results of Ref. [15] translated to the scales of our operators:

$$
\begin{aligned}
& \text { S - operators: } \Lambda_{S_{d d, X Y}}, \Lambda_{S_{u u, X Y}} \geq 5.8 \mathrm{TeV}, \Lambda_{S_{s s, X Y}} \geq 5 \mathrm{TeV} \text {, } \\
& \Lambda_{S_{s d, X Y}} \geq 1.9 \mathrm{TeV}, \Lambda_{S_{b d, X Y}} \geq 4.7 \mathrm{TeV}, \\
& \text { V-operators: } \Lambda_{V_{d d, X Y}}, \Lambda_{S_{u u, X Y}} \geq 5.7 \mathrm{TeV} \text {, } \\
& \Lambda_{V_{s s, X Y}} \geq 4.8 \mathrm{TeV}, \Lambda_{V_{s d, X Y}} \geq 1.8 \mathrm{TeV} \\
& \Lambda_{V_{b d, X Y}} \geq 4.1 \mathrm{TeV} \text {. }
\end{aligned}
$$

As seen from Tables XII and XIV, the DIS $\mu-\tau$ conversion on nuclei (1) covers a much wider set of the quark bilinears, providing limits on $\Lambda_{I_{i f, X Y}}$, where $i, f=u, d, s, c, b$ and
$I=S, V, T$. Our limits shown in Tables XI-XIV have been derived from the expected sensitivity (27) of the NA64 experiment. We conclude that the limits from the decays (31)-(32) on the set of $\Lambda$ in (32)-(33) are typically about factor stronger than ours, but there are missing limits on the operators with many other combinations of quark flavors, neither there are limits on the $\mathbf{T}$-operators, provided by the DIS $\mu-\tau$ conversion on nuclei (1).

The following comment on the scalar operators may be in order. It is known that the scalar LFV operators with heavy quarks can contribute LFV conversion via a triangle diagrams with two gluons legs immersed in a nucleus. This contribution is significant for the case of coherent nuclear $\mu-e$-conversion (see, for instance, Refs. [5,30]), being proportional to the nuclear mass. For deep inelastic LFV conversion, studied in our paper, this contribution is the part of the full contribution of the heavy-quark nucleon sea and is characterized by the following stages $g \rightarrow \bar{b}+b$, $b+\ell_{1} \rightarrow \ell_{2}+b, \bar{b}+b \rightarrow g$. In the inclusive setting of the experiment, which is the case of NA64, all the possible fates of the b quark are summed up after its interaction with the initial lepton, including channels with and without final $\bar{b}+b \rightarrow g$. Obviously, the channel with annihilation in the final state is subdominant in this sum.

Note that the $\mathbf{T}$-operators for some quark flavor combinations can be constrained by the decays (32). We have not found such limits in the literature and leave the study of this possibility for a future publication.

\section{POLARIZED LEPTON BEAM AND NUCLEAR TARGET}

Following Refs. [31,32], we consider the lepton conversion for specific choice of spin configurations of incoming beam and nuclear target. For the studied energies of incoming leptons we may safely neglect quark and lepton masses. As shown in Refs. [31,32] for $m_{N} \ll E_{\ell}$, the orientation of the nucleon spin $S$ is irrelevant, while there is a dependence on the helicity of the initial lepton $\lambda$.

The differential cross section of the $\ell-\tau$ conversion, taking into account the initial lepton helicity $\lambda$, can be written as

$$
\sigma_{\mathrm{pol}}(\ell+(A, Z) \rightarrow \tau+X)=\sum_{I, i f, X Y} \frac{Q_{I_{i f, X Y}^{A}}^{A}(\lambda)}{\Lambda_{I_{i f, X Y}}^{4}}
$$

with

$$
\begin{aligned}
Q_{I_{i f, X Y}}^{A}(\lambda)= & \frac{s}{64 \pi} \int_{0}^{1} d x \int_{0}^{1} d y\left[x f_{I, X Y}(y, \lambda) q_{i}^{A}(x, s x y)\right. \\
& \left.+x g_{I, X Y}(y, \lambda) \bar{q}_{f}^{A}(x, s x y)\right],
\end{aligned}
$$

where the functions $f_{I, X Y}(y, \lambda)$ and $g_{I, X Y}(y, \lambda)$ are given in Appendix. 
As seen from the Appendix, the choice of a particular helicity $\lambda$ of the initial lepton allows one to eliminate the contributions of certain operators to the $\ell-\tau$ flavor nondiagonal observables. In particular, the contribution of the operators $\mathcal{O}_{S_{i f, R R}^{\ell \tau}}^{\ell \tau}, \mathcal{O}_{S_{i f, R L}^{\ell \tau}}^{\ell \tau}, \mathcal{O}_{V_{i f, R R}}^{\ell \tau}, \mathcal{O}_{V_{i f, R L} \tau}^{\ell \tau}, \mathcal{O}_{T_{i f, R R} \tau}^{\ell \tau}$ is absent in quark-lepton subprocesses at $\lambda=+1$ and antiquark-lepton subprocesses at $\lambda=-1$. Correspondingly, the contribution of the operators $\mathcal{O}_{S_{i f, L L}^{\ell \tau}}^{\ell \tau}, \mathcal{O}_{S_{i f, L R}^{\ell \tau}}^{\ell}, \mathcal{O}_{V_{i f, L L}^{\ell \tau}}^{\ell \tau}, \mathcal{O}_{V_{i f, L R}^{\ell \tau}}^{\ell \tau}, \mathcal{O}_{T_{i f, L L}}^{\ell \tau}$ is absent in quark-lepton subprocesses at $\lambda=-1$ and antiquark-lepton subprocesses at $\lambda=+1$. Therefore, the experiments with polarized lepton beams could be useful for separating the contributions of different LFV operators and in this way to help us identify the underlying LFV theory.

Note that the averaging of the polarized cross section in Eq. (34) over $\lambda$ and $S$ reproduces our result for the unpolarized cross section (17),

$\sigma(\ell+(A, Z) \rightarrow \tau+X) \equiv \frac{1}{4} \sum_{\lambda, S} \sigma_{\mathrm{pol}}(\ell+(A, Z) \rightarrow \tau+X)$,

and in agreement with Ref. [32].

\section{SUMMARY}

We presented phenomenological analysis of the $e-\tau$ and $\mu-\tau$ conversion in electron (muon) scattering on fixed-target nuclei $(\mathrm{Pb}$ and $\mathrm{Fe})$. Our analysis is based on the effective four-fermion lepton-quark LFV operators linked to individual mass scales characterizing underlying renormalizable high-scale LFV physics. In the present paper, we have not considered its particular realization.

Using the expected sensitivity (27) of the NA64 experiment, we derived lower limits for the LFV mass scales of the effective operators and compared them with the corresponding limits existing in the literature, derived from the LFV decays. We have shown that the process of $e(\mu)+(A, Z) \rightarrow \tau+X$ is going to provide a plenty of new limits as yet nonexisting in the literature.

We predicted the spectrum of the final-state $\tau$-leptons helping one to assess the possibilities of discrimination of the signal from the typical backgrounds. We found that the mean $\tau$-lepton energy is significantly large than the required cutoff in the missing energy for all the effective operators (5)-(7). In our opinion this result supports the viability of searching for the process (1) with the NA64 experiment, which will able to come up with new limits presented in Tables XI-XIV, many of which do not yet exist in the literature. Currently, the NA64 searching for invisible decay of dark photon does not see background events with a large missing energy at the level $\sim 10^{-11}$ per $100 \mathrm{GeV}$ electron on $\mathrm{Pb}$ target [33]. This result can be used to extract limits on $\Lambda$ 's for the reaction of the $e-\tau$ conversion which will be reported elsewhere.
We also examined the possible advantages of polarized beams and targets and showed that they can help distinguish the contributions of different LFV operators. We hope our results will be useful for planning experiments searching for lepton flavor violation in accelerator experiments.

\section{ACKNOWLEDGMENTS}

This work was funded by the German Bundesministerium für Bildung und Forschung (BMBF) under Project No. 05P2015-ALICE at High Rate (BMBF-FSP 202): Jet- and fragmentation processes at ALICE and the parton structure of nuclei and structure of heavy hadrons, by Fondecyt (Chile) Grant No. 1150792, and by CONICYT (Chile) Ring ACT1406, PIA/Basal FB0821. This research was supported by the Tomsk State University competitiveness improvement program under Grant No. 8.1.07.2018.

\section{APPENDIX: POLARIZED AND UNPOLARIZED MATRIX ELEMENTS}

Here we give the definitions and the explicit forms of the functions $f_{I}$ and $g_{I}$ in Eqs. (15), (16), and (35) involved in the calculation of the square matrix elements of the operators in Eqs. (5)-(7).

For the case of unpolarized $\ell-\tau$ conversion, we have

$$
\begin{aligned}
& f_{S, L L}(y)=\operatorname{Tr}\left[P_{L} \not k P_{R} \not \ell^{\prime}\right] \operatorname{Tr}\left[P_{L} \not \not^{\prime} P_{R} \not ̋\right]=y^{2}, \\
& f_{S, R R}(y)=\operatorname{Tr}\left[P_{R} \not k P_{L} \not \ell^{\prime}\right] \operatorname{Tr}\left[P_{R} \not \not^{\prime} P_{L \not \supset}\right]=y^{2}, \\
& f_{S, L R}(y)=\operatorname{Tr}\left[P_{L} \not k P_{R} \not k^{\prime}\right] \operatorname{Tr}\left[P_{R} \not p^{\prime} P_{L} \not \not\right]=y^{2},
\end{aligned}
$$

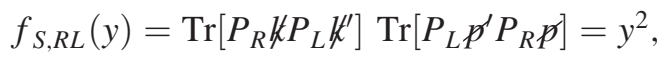

$$
\begin{aligned}
& f_{V, L L}(y)=\operatorname{Tr}\left[\gamma^{\mu} P_{L} \not k \gamma^{\nu} P_{L} \not k^{\prime}\right] \operatorname{Tr}\left[\gamma_{\mu} P_{L} \not \not \gamma_{\nu} P_{L} \not \not^{\prime}\right]=4, \\
& f_{V, R R}(y)=\operatorname{Tr}\left[\gamma^{\mu} P_{R} \not k \gamma^{\nu} P_{R} \not k^{\prime}\right] \operatorname{Tr}\left[\gamma_{\mu} P_{R} \not \not \gamma_{\nu} P_{R} \not p^{\prime}\right]=4 \text {, } \\
& f_{V, L R}(y)=\operatorname{Tr}\left[\gamma^{\mu} P_{L} \not k \gamma^{\nu} P_{L} \not k^{\prime \prime}\right] \operatorname{Tr}\left[\gamma_{\mu} P_{R} \not \gamma_{\nu} P_{R} \not \not^{\prime}\right]=4(1-y)^{2} \text {, } \\
& f_{V, R L}(y)=\operatorname{Tr}\left[\gamma^{\mu} P_{R} \not \gamma^{\nu} P_{R} \not k^{\prime}\right] \operatorname{Tr}\left[\gamma_{\mu} P_{L} \not \gamma_{\nu} P_{L} \not p^{\prime}\right]=4(1-y)^{2} \text {, }
\end{aligned}
$$

$$
\begin{aligned}
f_{T, L L}(y) & =\operatorname{Tr}\left[\sigma^{\mu \nu} P_{L} \not k P_{R} \sigma^{\alpha \beta} \not \ell^{\prime}\right] \operatorname{Tr}\left[\sigma_{\mu \nu} P_{L} \not p P_{R} \sigma_{\alpha \beta} \not p^{\prime}\right] \\
& =16(2-y)^{2}, \\
f_{T, R R}(y) & =\operatorname{Tr}\left[\sigma^{\mu \nu} P_{R} \not k P_{L} \sigma^{\alpha \beta} \not \not^{\prime}\right] \operatorname{Tr}\left[\sigma_{\mu \nu} P_{R} \not P P_{L} \sigma_{\alpha \beta} \not p^{\prime}\right] \\
& =16(2-y)^{2}
\end{aligned}
$$

and

$$
\begin{aligned}
& g_{S, L L}(y)=\operatorname{Tr}\left[P_{L} \not k P_{R} \not k^{\prime}\right] \operatorname{Tr}\left[P_{R} \not p^{\prime} P_{L} \not p\right]=y^{2}, \\
& g_{S, R R}(y)=\operatorname{Tr}\left[P_{R} \not k P_{L} \not k^{\prime}\right] \operatorname{Tr}\left[P_{L} \not p^{\prime} P_{R} \not \not\right]=y^{2}, \\
& g_{S, L R}(y)=\operatorname{Tr}\left[P_{L} \not k P_{R} \not k^{\prime}\right] \operatorname{Tr}\left[P_{L} \not p^{\prime} P_{R} \not p\right]=y^{2}, \\
& g_{S, R L}(y)=\operatorname{Tr}\left[P_{R} \not k P_{L} \not k^{\prime}\right] \operatorname{Tr}\left[P_{R} \not p^{\prime} P_{L} \not \not\right]=y^{2},
\end{aligned}
$$




$$
\begin{aligned}
& g_{V, L L}(y)=\operatorname{Tr}\left[\gamma^{\mu} P_{L} \not \gamma^{\nu} P_{L} \not \not^{\prime}\right] \operatorname{Tr}\left[\gamma_{\mu} P_{L} \not p^{\prime} \gamma_{\nu} P_{L \not p}\right]=4(1-y)^{2}, \\
& g_{V, R R}(y)=\operatorname{Tr}\left[\gamma^{\mu} P_{R} \not k \gamma^{\nu} P_{R} \not k^{\prime}\right] \operatorname{Tr}\left[\gamma_{\mu} P_{R} \not p^{\prime} \gamma_{\nu} P_{R} \not p\right]=4(1-y)^{2} \text {, } \\
& \left.g_{V, L R}(y)=\operatorname{Tr}\left[\gamma^{\mu} P_{L} \not k \gamma^{\nu} P_{L} \not k^{\prime}\right] \operatorname{Tr}\left[\gamma_{\mu} P_{R} \not p^{\prime} \gamma_{\nu} P_{R} \not\right]\right]=4 \text {, } \\
& g_{V, R L}(y)=\operatorname{Tr}\left[\gamma^{\mu} P_{R} \not k \gamma^{\nu} P_{R} \not k^{\prime}\right] \operatorname{Tr}\left[\gamma_{\mu} P_{L} \not p^{\prime} \gamma_{\nu} P_{L} \not p\right]=4 \text {, } \\
& g_{T, L L}(y)=\operatorname{Tr}\left[\sigma^{\mu \nu} P_{L} \not k \sigma^{\alpha \beta} P_{R} \not k^{\prime}\right] \operatorname{Tr}\left[\sigma_{\mu \nu} P_{L} \not P^{\prime} \sigma_{\alpha \beta} P_{R} \not p\right]=16(2-y)^{2}, \\
& g_{T, R R}(y)=\operatorname{Tr}\left[\sigma^{\mu \nu} P_{R} \not k \sigma^{\alpha \beta} P_{L} \not k^{\prime}\right] \operatorname{Tr}\left[\sigma_{\mu \nu} P_{R} \not{ }^{\prime} \sigma_{\alpha \beta} P_{L} \not p\right]=16(2-y)^{2} .
\end{aligned}
$$

In the case of a polarized lepton beam and target, the corresponding functions are

$$
\begin{aligned}
& f_{S, L L}(y, \lambda)=\operatorname{Tr}\left[P_{L} \not k\left(1+\gamma_{5} \lambda\right) P_{R} \not k^{\prime}\right] \operatorname{Tr}\left[P_{L} \not p P_{R} \not \not^{\prime}\right]=y^{2}(1+\lambda), \\
& f_{S, R R}(y, \lambda)=\operatorname{Tr}\left[P_{R} \not k\left(1+\gamma_{5} \lambda\right) P_{L} \not k^{\prime \prime}\right] \operatorname{Tr}\left[P_{R} \not P_{L} \not \not^{\prime}\right]=y^{2}(1-\lambda), \\
& f_{S, L R}(y, \lambda)=\operatorname{Tr}\left[P_{L} \not k\left(1+\gamma_{5} \lambda\right) P_{R} \not k^{\prime}\right] \operatorname{Tr}\left[P_{R} \not p P_{L} \not \not^{\prime}\right]=y^{2}(1+\lambda), \\
& f_{S, R L}(y, \lambda)=\operatorname{Tr}\left[P_{R} \not k\left(1+\gamma_{5} \lambda\right) P_{L} \not k^{\prime \prime}\right] \operatorname{Tr}\left[P_{L} \not p P_{R} \not \not^{\prime}\right]=y^{2}(1-\lambda), \\
& f_{V, L L}(y, \lambda)=\operatorname{Tr}\left[\gamma^{\mu} P_{L} \not k\left(1+\gamma_{5} \lambda\right) \gamma^{\nu} P_{L} \not k^{\prime}\right] \operatorname{Tr}\left[\gamma_{\mu} P_{L} \not \not \gamma_{\nu} P_{L} \not \not^{\prime}\right]=4(1+\lambda), \\
& f_{V, R R}(y, \lambda)=\operatorname{Tr}\left[\gamma^{\mu} P_{R} \not k\left(1+\gamma_{5} \lambda\right) \gamma^{\nu} P_{R} \not k^{\prime \prime}\right] \operatorname{Tr}\left[\gamma_{\mu} P_{R} \not \gamma_{\nu} P_{R} \not \not^{\prime}\right]=4(1-\lambda), \\
& f_{V, L R}(y, \lambda)=\operatorname{Tr}\left[\gamma^{\mu} P_{L} \not k\left(1+\gamma_{5} \lambda\right) \gamma^{\nu} P_{L} \not \ell^{\prime}\right] \operatorname{Tr}\left[\gamma_{\mu} P_{R} \not \gamma_{\nu} P_{R} \not \not^{\prime}\right]=4(1-y)^{2}(1+\lambda) \text {, } \\
& f_{V, R L}(y, \lambda)=\operatorname{Tr}\left[\gamma^{\mu} P_{R} \not k\left(1+\gamma_{5} \lambda\right) \gamma^{\nu} P_{R} \not k^{\prime}\right] \operatorname{Tr}\left[\gamma_{\mu} P_{L} \not \gamma_{\nu} P_{L} \not p^{\prime}\right]=4(1-y)^{2}(1-\lambda) \text {, } \\
& f_{T, L L}(y, \lambda)=\operatorname{Tr}\left[\sigma^{\mu \nu} P_{L} \not k\left(1+\gamma_{5} \lambda\right) \sigma^{\alpha \beta} P_{R} \not k^{\prime \prime}\right] \operatorname{Tr}\left[\sigma_{\mu \nu} P_{L} \not p \sigma_{\alpha \beta} P_{R} \not \not^{\prime}\right]=16(2-y)^{2}(1+\lambda), \\
& f_{T, R R}(y, \lambda)=\operatorname{Tr}\left[\sigma^{\mu \nu} P_{R} \not k\left(1+\gamma_{5} \lambda\right) \sigma^{\alpha \beta} P_{L} \not k^{\prime \prime}\right] \operatorname{Tr}\left[\sigma_{\mu \nu} P_{R} \not \sigma_{\alpha \beta} P_{L} \not \not^{\prime}\right]=16(2-y)^{2}(1-\lambda) \text {, }
\end{aligned}
$$

and

$$
\begin{aligned}
& g_{S, L L}(y, \lambda)=\operatorname{Tr}\left[P_{L} \not k\left(1-\gamma_{5} \lambda\right) P_{R} \not k^{\prime}\right] \operatorname{Tr}\left[P_{L} \not P^{\prime} P_{R} \not \partial\right]=y^{2}(1-\lambda), \\
& g_{S, R R}(y, \lambda)=\operatorname{Tr}\left[P_{R} \not k\left(1-\gamma_{5} \lambda\right) P_{L} \not k^{\prime}\right] \operatorname{Tr}\left[P_{R} \not \not^{\prime} P_{L} \not p\right]=y^{2}(1+\lambda), \\
& g_{S, L R}(y, \lambda)=\operatorname{Tr}\left[P_{L} \not k\left(1-\gamma_{5} \lambda\right) P_{R} \not k^{\prime}\right] \operatorname{Tr}\left[P_{R} \not P^{\prime} P_{L} \not p\right]=y^{2}(1-\lambda) \text {, } \\
& g_{S, R L}(y, \lambda)=\operatorname{Tr}\left[P_{R} \not k\left(1-\gamma_{5} \lambda\right) P_{L} \not k^{\prime}\right] \operatorname{Tr}\left[P_{L} \not \not^{\prime} P_{R} \not p\right]=y^{2}(1+\lambda) \text {, } \\
& \left.g_{V, L L}(y, \lambda)=\operatorname{Tr}\left[\gamma^{\mu} P_{L} \not k\left(1-\gamma_{5} \lambda\right) \gamma^{\nu} P_{L} \not k^{\prime \prime}\right] \operatorname{Tr}\left[\gamma_{\mu} P_{L} \not \not^{\prime} \gamma_{\nu} P_{L} \not \not\right]\right]=4(1-y)^{2}(1-\lambda), \\
& g_{V, R R}(y, \lambda)=\operatorname{Tr}\left[\gamma^{\mu} P_{R} \not k\left(1-\gamma_{5} \lambda\right) \gamma^{\nu} P_{R} \not k^{\prime}\right] \operatorname{Tr}\left[\gamma_{\mu} P_{R} \not \not^{\prime} \gamma_{\nu} P_{R} \not \not\right]=4(1-y)^{2}(1+\lambda) \text {, } \\
& g_{V, L R}(y, \lambda)=\operatorname{Tr}\left[\gamma^{\mu} P_{L} \not k\left(1-\gamma_{5} \lambda\right) \gamma^{\nu} P_{L} \not k^{\prime}\right] \operatorname{Tr}\left[\gamma_{\mu} P_{R} \not \not^{\prime} \gamma_{\nu} P_{R} \not \gamma\right]=4(1-\lambda) \text {, } \\
& g_{V, R L}(y, \lambda)=\operatorname{Tr}\left[\gamma^{\mu} P_{R} \not k\left(1-\gamma_{5} \lambda\right) \gamma^{\nu} P_{R} \not k^{\prime}\right] \operatorname{Tr}\left[\gamma_{\mu} P_{L} \not p^{\prime} \gamma_{\nu} P_{L} \not p\right]=4(1+\lambda) \text {, } \\
& g_{T, L L}(y, \lambda)=\operatorname{Tr}\left[\sigma^{\mu \nu} P_{L} \not k\left(1-\gamma_{5} \lambda\right) \sigma^{\alpha \beta} P_{R} \not k^{\prime}\right] \operatorname{Tr}\left[\sigma_{\mu \nu} P_{L} \not \not^{\prime} \sigma_{\alpha \beta} P_{R} \not \not\right]=16(2-y)^{2}(1-\lambda), \\
& \left.g_{T, R R}(y, \lambda)=\operatorname{Tr}\left[\sigma^{\mu \nu} P_{R} \not k\left(1-\gamma_{5} \lambda\right) \sigma^{\alpha \beta} P_{L} \not k^{\prime}\right] \operatorname{Tr}\left[\sigma_{\mu \nu} P_{R} \not \not^{\prime} \sigma_{\alpha \beta} P_{L} \not \not\right]\right]=16(2-y)^{2}(1+\lambda) \text {, }
\end{aligned}
$$

where $\lambda$ is the helicity of the initial lepton. 
[1] S. Chekanov et al. (ZEUS Collaboration), Phys. Rev. D 65, 092004 (2002).

[2] S. N. Gninenko, M. M. Kirsanov, N. V. Krasnikov, and V. A. Matveev, Mod. Phys. Lett. A 17, 1407 (2002).

[3] A. Faessler, T. S. Kosmas, S. Kovalenko, and J. D. Vergados, Nucl. Phys. B587, 25 (2000).

[4] T. S. Kosmas, S. Kovalenko, and I. Schmidt, Phys. Lett. B 511, 203 (2001).

[5] T. S. Kosmas, S. Kovalenko, and I. Schmidt, Phys. Lett. B 519, 78 (2001).

[6] F. Simkovic, A. Faessler, S. Kovalenko, and I. Schmidt, Phys. Rev. D 66, 033005 (2002).

[7] F. Simkovic, V. E. Lyubovitskij, T. Gutsche, A. Faessler, and S. Kovalenko, Phys. Lett. B 544, 121 (2002).

[8] A. Faessler, T. Gutsche, S. Kovalenko, V. E. Lyubovitskij, I. Schmidt, and F. Simkovic, Phys. Lett. B 590, 57 (2004).

[9] A. Faessler, T. Gutsche, S. Kovalenko, V. E. Lyubovitskij, I. Schmidt, and F. Simkovic, Phys. Rev. D 70, 055008 (2004).

[10] A. Faessler, T. Gutsche, S. Kovalenko, V. E. Lyubovitskij, and I. Schmidt, Phys. Rev. D 72, 075006 (2005).

[11] T. Gutsche, J. C. Helo, S. Kovalenko, and V. E. Lyubovitskij, Phys. Rev. D 81, 037702 (2010).

[12] T. Gutsche, J. C. Helo, S. Kovalenko, and V. E. Lyubovitskij, Phys. Rev. D 83, 115015 (2011).

[13] M. Gonzalez, T. Gutsche, J. C. Helo, S. Kovalenko, V. E. Lyubovitskij, and I. Schmidt, Phys. Rev. D 87, 096020 (2013).

[14] S. N. Gninenko, M. M. Kirsanov, N. V. Krasnikov, V. A. Matveev, P. Nedelec, D. Sillou, and M. Sher, CERN, Report Nos. CERN-SPSC-2004-016, SPSC-EOI-004.

[15] D. Black, T. Han, H. J. He, and M. Sher, Phys. Rev. D 66, 053002 (2002).

[16] M. Sher and I. Turan, Phys. Rev. D 69, 017302 (2004).
[17] A. Brignole and A. Rossi, Nucl. Phys. B701, 3 (2004).

[18] S. Kanemura, Y. Kuno, M. Kuze, and T. Ota, Phys. Lett. B 607, 165 (2005).

[19] P. Paradisi, J. High Energy Phys. 08 (2006) 047.

[20] M. Gonderinger and M. J. Ramsey-Musolf, J. High Energy Phys. 11 (2010) 045; 05 (2012) 047(E).

[21] A. Bolanos, A. Fernandez, A. Moyotl, and G. TavaresVelasco, Phys. Rev. D 87, 016004 (2013).

[22] F. Cei and D. Nicolo, Adv. High Energy Phys. 2014, 282915 (2014).

[23] A. Abada, V. De Romeri, J. Orloff, and A. M. Teixeira, Eur. Phys. J. C 77, 304 (2017).

[24] M. Takeuchi, Y. Uesaka, and M. Yamanaka, Phys. Lett. B 772, 279 (2017).

[25] D. Banerjee et al. (NA64 Collaboration), Phys. Rev. Lett. 118, 011802 (2017).

[26] J. Gao, M. Guzzi, J. Huston, H.-L. Lai, Z. Li, P. Nadolsky, J. Pumplin, D. Stump, and C.-P. Yuan, Phys. Rev. D 89, 033009 (2014).

[27] P. M. Nadolsky, H. L. Lai, Q. H. Cao, J. Huston, J. Pumplin, D. Stump, W. K. Tung, and C.-P. Yuan, Phys. Rev. D 78, 013004 (2008).

[28] H. Bethe and W. Heitler, Proc. R. Soc. A 146, 83 (1934).

[29] Y.S. Tsai, Rev. Mod. Phys. 46, 815 (1974); 49, 421(E) (1977).

[30] V. Cirigliano, R. Kitano, Y. Okada, and P. Tuzon, Phys. Rev. D 80, 013002 (2009).

[31] J. A. Bartelski, Acta Phys. Pol. B 10, 923 (1979).

[32] M. Anselmino, P. Gambino, and J. Kalinowski, Z. Phys. C 64, 267 (1994).

[33] D. Banerjee et al. (NA64 Collaboration), Phys. Rev. D 97, 072002 (2018). 